\title{
Consistência interna e fatorial do Inventário Multifatorial de Coping para Adolescentes
}

\author{
Marcos Alencar Abaide Balbinotti ${ }^{1}$ \\ Marcus Levi Lopes Barbosa \\ Daniela Wiethaeuper
}

\begin{abstract}
Resumo
Coping é um construto multidimensional relativo às formas como as pessoas lidam com situações estressantes. Diversas pesquisas assinalam a importância dessas "respostas de enfrentamento". Este estudo visa verificar os índices de consistência interna e fatorial confirmatórios do Inventário Multifatorial de Coping para Adolescentes (IMCA-43). Assim, a coleta de dados foi realizada mediante aplicações coletivas, em sala de aula, em uma amostra de 285 estudantes do ensino fundamental e médio, de ambos os sexos e com idades variando de 13 a 18 anos. Os resultados dos índices alfa de Cronbach $(0,71$ a 0,89$)$ foram satisfatórios. A adequação aos modelos tridimensional $\left(\chi^{2} / \mathrm{gl}=2,85 ; \mathrm{GFI}=0,757\right.$; AGFI $\left.=0,724 ; \mathrm{RMSEA}=0,081\right)$ tetradimensional $\left(\chi^{2} / \mathrm{gl}=2,44 ; \mathrm{GFI}=0,724 ; \mathrm{AGFI}=\right.$ $0,695$; RMSEA $=0,071)$ e pentadimensional $\left(\chi^{2} / \mathrm{gl}=2,32\right.$; GFI $=0,750$; AGFI $=0,723$; RMSEA $\left.=0,068\right)$ é pouco recomendada. Os resultados indicam serem necessárias pesquisas continuadas a fim de melhorar certas qualidades métricas deste instrumento.
\end{abstract}

Palavras-chave: Coping; Avaliação; Estudantes; Validação; Psicometria.

\section{Reliability and confirmatory factorial analysis of the Multifactor Coping for Adolescents Inventory with Brazilian students}

\begin{abstract}
Coping is a multidimensional concept concerning how people face and deal with stressful situations. Researches have been showing the importance of these forms of behavior responses. This study aimed to measure the internal consistency of the Multifactorial Inventory of Coping for Adolescents (IMCA-43) and evaluate the fit of the model through confirmatory factorial analysis. A sample of 285 students of Intermediate and High School, of both sexes, with ages ranging from 13 to 18 years old was used. The data was colected colectivelly in their classrooms. The results of the Cronbach alfa $(0,71$ to 0,89$)$ were satisfactory. The index of adequacy for the tri-dimensional model $\left(\chi^{2} / \mathrm{gl}=2,85 ; \mathrm{GFI}=0,757 ; \mathrm{AGFI}=0,724 ; \mathrm{RMSEA}=0,081\right)$, tetra-dimensional $\left(\chi^{2} / \mathrm{gl}=2,44 ; \mathrm{GFI}=0,724 ;\right.$ AGFI $=0,695 ;$ RMSEA $=0,071)$ and penta-dimensional $\left(\chi^{2} / \mathrm{gl}=2,32 ; \mathrm{GFI}=0,750 ; \mathrm{AGFI}=0,723\right.$; RMSEA $\left.=0,068\right)$ are non-recommended for the fit of the model. The results indicate that new researches are necessary to improve the metric qualities of the instrument.
\end{abstract}

Keywords: Coping; Measure; Students; Validation; Psychometric.

\section{Introdução}

Esta pesquisa visa explorar qualidades métricas do Inventário Multifatorial de Coping para Adolescentes (IMCA-43) (Antoniazzi, 2000), com base em novos dados colhidos na realidade brasileira em uma amostra de estudantes de duas escolas públicas, de ensino fundamental e médio, do sul do Brasil. Ainda, pretendem-se explorar possíveis semelhanças e diferenças em alguns dados obtidos com essa amostra e as de outros estudos. Para melhor responder a esses objetivos, apresentam-se, inicialmente, aspectos referentes ao plano teórico relativo ao conceito de coping e, posteriormente, os aspectos referentes ao plano empírico, também relativos a este mesmo construto, apresentando alguns resultados importantes de pesquisa onde, de certa forma, já foram exploradas estas e outras qualidades métricas desse mesmo inventário.

\section{Coping no plano teórico}

No plano teórico (Lazarus \& Folkman, 1984), coping é o conjunto de esforços cognitivos e comportamentais, ou estratégias de enfrentamento, realizados pelo indivíduo com o objetivo de lidar com as demandas, internas e externas, que são por ele avaliadas como sobrecarregando ou excedendo seus recursos pessoais. Originalmente, partindo de pressupostos da psicanálise, coping foi concebido como um correlato dos mecanismos de defesa (Vaillant, 1994). Na década de 60, estes mecanismos (coping) passaram a ser vistos como um processo flexível e proposital, adequados à realidade e

${ }^{1}$ Endereço para correspondência:

Rua Luzitana 1.398/501 - Bairro Higienópolis - 90520-080 - Porto Alegre-RS - Tel: (51) 3342-3852 - Fax: (51) 3337-1547

E-mail:mbalbinotti@terra.com.broubalbinotti@unisinos.br 
orientados para o futuro, ou seja, estratégias conscientes, usadas pelos indivíduos diante de situações de estresse (Parker \& Endler, 1996). Esta nova concepção pressupõe que tais estratégias são orientadas conforme a especificidade do contexto, variando em razão das particularidades da situação estressante, do tempo e da quantidade de estresse envolvido na situação (Lazarus \& Folkman, 1984). Mais recentemente, baseados nas evidências da estabilidade de determinadas respostas de coping no tempo e, através de situações semelhantes enfrentadas pelos indivíduos, têm se buscado convergências entre o conceito de coping e o de personalidade (Costa, Somerfield \& McCrae, 1996).

Holahan, Moos e Shaefer (1996) definem coping como um "traço", considerando os diferentes estilos de coping como disposições de personalidade que transcendem a influência de contextos situacionais ou de tempo, ou seja, estabelecem esta noção de estabilidade. O modelo, por eles adotado, caracteriza-se por relacionar as diferentes respostas ou estratégias de coping ao desempenho de tarefas adaptativas por parte dos indivíduos. Por exemplo, as estratégias de evitação são compostas por respostas de coping onde o problema é colocado a distância até que o tempo ou outro fator modifique a situação estressante. Consideradas mais adaptativas, as estratégias de aproximacão são aquelas onde o indivíduo tenta lidar diretamente com o problema, reavaliando a situação com ajuda de um suporte social (Holahan \& Moos, 1985).

Mesmo que ainda relativamente pouco explorado no Brasil (Antoniazzi, Dell'Aglio \& Bandeira, 1998; Antoniazzi, 2000; Dell'Aglio, 2000; Dell'Aglio \& Hutz, 2002; Gimenes, 1997; Seidl, Tróccoli \& Zannon, 2001), o conceito de coping tem tido considerável atenção na literatura internacional. Recentemente, têm sido teoricamente hipotetizadas as relações deste construto com saúde (Endler, Summerfledt \& Parker, 1998; Gayton, 2002; Wearden, Cook \& Vaughan-Jones, 2003), personalidade (Lefkowitz, 2003; Wang \& Wang, 2003; Willebrand, 2003; Windover, 2002), auto-estima (Darnall, 2002; Johnson, 2004; Lee, 2004), adaptabilidade e vulnerabilidade social (Endler \& Parker, 1990; Lefkowitz, 2003; Weisz, McCabe \& Denning, 1994), depressão (Croyle e colaboradores 2003; Hussain \& Cochrane, 2003; Tucker, Brust \& Richardson, 2002; Ying, Wen-bin \& Guo-qiang, 2003), dentre muitos outros.

\section{Coping no plano empírico}

Estudos tratam da elaboração de modelos explicativos do comportamento de coping ou da verificação da aplicabilidade empírica desses modelos (Altshuler \& Ruble, 1989; Band \& Weisz, 1988;
Holahan e colaboradores, 1996; Lazarus \& Folkman, 1984). De forma complementar, outras pesquisas têm buscado entender certas nuances do construto. Por exemplo, Lopez e Little (1996) têm explorado esse construto verificando a influência do gênero na escolha de estratégias de coping, uma vez que meninos e meninas são socializados de variadas formas. As diferenças verificadas são entendidas como o resultado da socialização das meninas para o uso de estratégias prósociais, enquanto os meninos são socializados para serem independentes e competitivos.

Outras pesquisas têm buscado as relações entre o comportamento de coping e a idade (Compas, Banez, Malcarne \& Worsham, 1991; Heckhausen \& Schulz, 1995). Elas sugerem que esse comportamento está relacionado ao desenvolvimento cognitivo e à capacidade de auto-regulação das emoções.

No plano empírico, recentemente tem-se notado um crescente número de estudos com respeito à validade do construto coping. Alguns deles são iniciativas verificadas especialmente nos Estados Unidos, Canadá, Espanha e Brasil, com o objetivo de mensurar ou verificar a validade transcultural das medidas do comportamento de coping (Amirkhan, 1990; Carver, Scheier \& Weintraub, 1989; Endler \& Parker, 1990, 1999; Folkman, Lazarus, Dunkel-Schetter, DeLongis \& Gruen, 1986; Folkman \& Lazarus, 1985; RodríguezMarín, Terol, López-Roig \& Pastor, 1992; Vitalino, Russo, Carr, Maiuro \& Becker, 1985).

\section{Inventários de coping}

Para melhor responder às necessidades teóricas de hipotetizações concernentes às relações apresentadas diversos instrumentos têm sido desenvolvidos para avaliar coping. Alguns deles são o Ways of Coping Checklist - WCC (Folkman \& Lazarus, 1980), que foi revisado em 1985 por Vitalino e colaboradores, o Ways of Coping Questionnaire Revised - WOCQ-R (Folkman e colaboradores 1986), o Ways of Coping Questionnaire WCQ (Parker, Endler \& Bagby, 1993; Schwarzer \& Schwarzer, 1996), o COPE Inventory (Carver e colaboradores, 1989) e o Coping Strategy Indicator - CSI (Amirkhan, 1990).

No Brasil ainda há uma carência de instrumentos adaptados, válidos e fidedignos para avaliação de coping na população. Nota-se, porém, nos últimos anos, um crescente interesse pelo conceito e um acréscimo no número de estudos com este objetivo. Em 1996, Savóia, Santana e Mejias publicaram uma adaptação do Ways of Coping Checklist - WCC de Folkmam e Lazarus. Em 1997, Gimenes e Queiroz publicaram a tradução e adaptação (tradução reversa e 
análise semântica) de uma versão da Ways of Coping Checklist de Vitaliano e colaboradores (1985) que resultou na Escala Modos de Enfrentamento de Problemas. Mais recentemente, Seidl e colaboradores (2001) fizeram um estudo da estrutura fatorial da mesma escala. Tróccoli e colaboradores (Pinheiro, Tamayo \& Tróccoli, 2000) têm trabalhado na tradução e adaptação da versão reduzida do Constructive Thinking Inventory (CTI-S), desenvolvida por Epstein (1990). No entanto, a literatura indica a presença de fraquezas metodológicas como, por exemplo, medidas inadequadas do ponto de vista psicométrico, estruturas fatoriais pouco investigadas e ausência de validação de instrumentos para populações específicas (Clark, Bormann, Cropanzano \& James, 1995). Como tentativa de solução desses problemas, Antoniasi (2000) desenvolveu o Inventário Multifatorial de Coping para Adolescentes (IMCA-43).

\section{Inventário Multifatorial de Coping para Adolescentes (IMCA-43)}

Antoniasi (2000) desenvolveu o IMCA-43 a fim de obter uma medida válida desse construto em adolescentes. Trata-se de uma escala auto-aplicável de 43 itens distribuídos em 4 fatores: ações diretas, apoio social, negação e autocontrole. O Inventário Multifatorial de Coping para Adolescentes foi elaborado com base em 77 itens obtidos em estudos anteriores e alguns itens elaborados para completar as dimensões que apresentaram um número reduzido de itens. As dimensões completadas foram as subcategorias do modelo Aproximação-Evitação de Holahan e colaboradores (1996), que são, por um lado, as categorias de aproximação: ações diretas, reavaliação, apoio social e autocontrole, e, por outro lado, as categorias de evitação: inibição da ação, distração, negação e ações agressivas.

Esses itens serviram de instrumento na coleta de dados, sendo respondidos por 783 estudantes (15 a 30 anos; $\mathrm{M}=18$ ) em uma escala Likert de 5 pontos, que vai de "nunca faço isso" a "sempre faço isso". A solução fatorial encontrada selecionou 43 itens em quatro fatores que explicaram $39,7 \%$ da variância total.

O primeiro fator foi denominado ações diretas $(\alpha=0,86)$ e agrupou 14 itens com conteúdos próprios das categorias ações diretas e reavaliação. Apresentou um eigenvalue de 7,33 e explicou 16,6\% da variância total. $\mathrm{O}$ segundo fator foi chamado de apoio social $(\alpha=0,81)$ e agregou 8 itens com conteúdo próprio dessa categoria. O fator apoio social apresentou eigenvalue de 4,83 e explicou 10,99\% da variância total. O terceiro fator, denominado Psico-USF, v. 11, n. 2, p. 175-183, jul./ dez. 2006 negação $(\alpha=0,76)$, agrupou 11 itens com as categorias de coping negação, distração e inibição da ação, apresentando um eigenvalue de 2,79 e explicando $6,35 \%$ da variância total. O quarto fator, chamado autocontrole $(\alpha=0,81)$, agrupou 10 itens com conteúdos de autocontrole e ações agressivas. O autovalor foi de 2,5 e a porcentagem explicada da variância total foi de 5,68\%.

Para fins deste estudo, verificou-se que, recentemente, foram realizados dois estudos empíricos utilizando IMCA-43. O primeiro deles verificou a fidedignidade e a validade fatorial exploratória da escala (Barbosa \& Balbinotti, 2003). Os resultados obtidos com uma amostra de 267 sujeitos confirmaram a fidedignidade das dimensões da escala (ações diretas: $\alpha=0,86$; apoio social: $\alpha=$ 0,76; negação: $\alpha=0,75$; autocontrole: $\alpha=0,70)$. Entretanto, a análise fatorial exploratória detectou problemas relacionados ao fator autocontrole, que reúne itens com conteúdos contraditórios (autocontrole e ações agressivas). Na verdade dos 10 itens que deveriam saturar neste fator, apenas 4 saturaram de forma importante onde se esperava (saturações fatoriais superiores a 0,30); os demais itens (aqueles cujo conteúdo era de autocontrole) saturaram na dimensão ações diretas.

O segundo estudo utilizando o IMCA-43 empregou uma amostra de 189 alunos de escolas públicas da Grande Porto Alegre (Balbinotti, Barbosa \& Wiethaeuper, no prelo). Da mesma forma que no estudo anterior, a consistência interna das escalas obtidas através do coeficiente alfa de Cronbach foi satisfatória $(0,72$ a 0,87$)$. No que diz respeito à validade fatorial da escala medida por meio de uma análise fatorial exploratória (mesmo método do estudo original), problemas como itens saturando de forma importante (saturações fatoriais superiores a 0,30) em mais de um fator ou não saturando de forma importante (saturações fatoriais inferiores a 0,30) em nenhum fator e, o mais importante, a nãoconfirmação do quarto fator (autocontrole) foi observada. Os resultados desses estudos levantam dúvidas sobre a adequação da solução fatorial encontrada pela autora e o modelo em quatro dimensões.

Partindo-se dos planos teóricos e empíricos apresentados anteriormente, foi possível formular a seguinte questão de pesquisa: "a partir de dados colhidos em uma amostra da população de estudantes, poder-se-ão encontrar índices de consistência interna e fatorial confirmatória que justifiquem o uso do IMCA-43?" Para bem responder essa questão, foram empregados procedimentos 
metodológicos, éticos e estatísticos, os quais serão apresentados a seguir.

\section{Método}

\section{Participantes}

A escolha da amostra foi por conveniência (não-aleatória), com o cuidado de evitar grupos de classes especiais. Os 285 estudantes com idade variando entre 13 e 18 anos $(M=15,09 ; \mathrm{DP}=1,31)$, sendo 132 $(46 \%)$ do sexo masculino e 153 (54\%) do sexo feminino, $150(52,6 \%)$ adolescentes estudantes do ensino fundamental ( $75=7^{\mathrm{a}}$ série; $75=8^{\mathrm{a}}$ série) e 135 $(47,4 \%)$ do ensino médio $\left(75=1^{\circ}\right.$ ano; $60=2^{\circ}$ ano $)$, estavam todos regularmente matriculados em escolas públicas de duas cidades da região metropolitana de Porto Alegre.

\section{Instrumentos}

Foram utilizados dois instrumentos: um Questionário Sócio-Demográfico Simples (QSDS), apenas para controle das variáveis sexo e idade, e o Inventário Multifatorial de Coping para Adolescentes (IMCA-43). O IMCA-43 (Antoniasi, 2000) é composto por 43 itens que avaliam 4 dimensões referentes a traços latentes do comportamento de coping: ações diretas (14 itens), apoio social (8 itens), negação (11 itens) e autocontrole (10 itens). Na aplicação do instrumento, os adolescentes foram convidados a lembrar de uma situação difícil pela qual tenham passado recentemente e a responder, em uma escala tipo Likert de cinco pontos, indo de 1 (nunca faço isso), a 5 (sempre faço isso), a freqüência com a qual utilizam as estratégias descritas nos itens. As qualidades psicométricas originais já estão referidas neste trabalho.

\section{Procedimento}

Inicialmente, contataram-se os coordenadores e diretores das escolas investigadas. O objetivo deste(s) primeiro(s) encontro(s) era obter a permissão livre e esclarecida para entrar nas salas de aula. Assim, entre 5 ou 7 dias anteriores à administração do inventário, todos os estudantes eram convidados pelo próprio professor a participar. Um dia foi marcado para ser aplicado um instrumento de pesquisa que buscaria informações a respeito da forma de lidar com situações difíceis. As aplicações foram realizadas coletivamente, em sala de aula, com um tempo aproximado de 20 minutos por aplicação. Não houve grupos maiores que 29 participantes por aplicação. O consentimento livre e esclarecido assinado foi obtido de todos e a confidencialidade foi assegurada.

\section{Resultados}

Para responder adequadamente à questão central desta pesquisa, procedeu-se à exploração dos escores obtidos pelo IMCA-43, segundo princípios norteadores comumente aceitos na literatura especializada (Angers, 1992; Bryman \& Cramer, 1999; Cronbach \& Meehl, 1955; Dassa, 1999; Nunnally, 1978; Pestana \& Gageiro, 2003; Reis, 2001; Taylor, Bagby \& Parker, 2003). Após os caminhos feitos apresentam-se, sucessiva e sistematicamente, os resultados do estudo da consistência interna da escala e da análise fatorial confirmatória (para o estudo da pesquisa da validade do inventário).

\section{Consistência interna}

Antes de se chegar às análises propriamente ditas do índice alfa de Cronbach obtido por esta amostra, foram conduzidas estatísticas preliminares que fundamentam este índice da consistência interna do IMCA-43. Assim, destaca-se que as médias encontradas para cada um dos 43 itens, estudados individualmente, variaram entre 1,94 e $3,85(\bar{X}=2,99)$; com desvios padrão entre 1,13 e 1,53 .

Tabela 1 - Análises descritivas: estatísticas de tendência central e dispersão

\begin{tabular}{ccccc}
\hline & & \multicolumn{3}{c}{ Análises descritivas } \\
\cline { 3 - 5 } Fator & Alfa & \multicolumn{3}{c}{ Tendência central (dispersão) } \\
\cline { 3 - 5 } & & $\bar{X}(\underline{\text { DP })})$ & $\begin{array}{c}\text { Mínimo } \\
\text { esperado/ } \\
\text { observado }\end{array}$ & $\begin{array}{c}\text { Máximo } \\
\text { esperado/ } \\
\text { observado }\end{array}$ \\
\hline Ações diretas & 0,89 & $45,91(11,57)$ & $14 / 14$ & $70 / 70$ \\
Apoio social & 0,74 & $22,16(6,61)$ & $8 / 8$ & $40 / 38$ \\
Negação & 0,74 & $33,28(7,78)$ & $11 / 12$ & $55 / 50$ \\
Autocontrole & 0,71 & $27,09(5,58)$ & $10 / 10$ & $50 / 44$ \\
\hline
\end{tabular}

Quanto às médias encontradas nas dimensões do instrumento (ver Tabela 1), observa-se que não há adesão aos limites (superior ou inferior). Os valores dos desvios padrão são adequados, já que em nenhum caso são superiores à metade do valor nominal das médias a que estão associados. De forma geral, as distribuições possuem valores observados idênticos ou muito próximos aos valores esperados.

No que diz respeito às correlações, a mediana das correlações item total foi moderada $(r=0,43)$. As correlações item dimensão apresentaram-se bastante satisfatórias: ações diretas (variaram de $r=0,46$ a $r=0,65$ ), apoio social (variaram de $r=0,37$ a $r=0,54$ ), negação (variaram de $r=0,30$ a $r=0,50$ ) e autocontrole (variaram 
de $r=0,20$ a $r=0,49$, com apenas dois itens apresentando correlações inferiores a 0,30 ).

O coeficiente alfa foi obtido a partir do SPSS 11,5, verificando-se a consistência interna do Inventário Multifatorial de Coping Para Adolescentes. Foi utilizado o modelo proposto por Cronbach e seus colaboradores (Cronbach, 1951, 1988, 1989, 1996; Cronbach \& Meehl, 1955; Cronbach, Rajaratnam \& Gleser, 1963). Garson (2005), Pestana e Gageiro (2003), Nunnally (1978) e Taylor e colaboradores (2003) recomendam que a adequação e a satisfatoriedade do coeficiente alfa sejam testadas usando-se os seguintes critérios: o coeficiente alfa recomendado deve ser superior a 0,70 , mas pode ser aceito quando superior a 0,60, dessa forma garantindo-se satisfatória consistência interna do instrumento.

Com base nesses dados preliminares apresentados, pode-se avaliar o coeficiente alfa para as dimensões da escala em estudo. Como se pode ver (Tabela 1), em todos os casos os resultados indicam satisfatórios índices de consistência interna para o IMCA43.

\section{Análise fatorial confirmatória}

O modelo de quatro fatores do IMCA-43 foi testado a partir do pacote AMOS 4.0, verificando-se sua adequabilidade (Berry, Poortinga, Segall \& Dasen, 1992). Foi usado o modelo proposto por Bagby, Taylor e Parker (1992), que considera que cada item deve aferir apenas um fator, diferenciando-se, portanto, do modelo fatorial exploratório (onde cada item apresenta saturações fatoriais nos diversos fatores com valores próprios superiores a 1).

Assim, partindo-se da hipotética associação entre as quatro dimensões do construto coping para a amostra de estudantes, um modelo fatorial oblíquo foi testado. Seguindo as recomendações de Cole (1987), Watkins (1989) e Briggs e Cheek (1986), a adequação do modelo fatorial confirmatório foi testada usando cinco critérios.

Critérios múltiplos foram utilizados, já que cada índice apresenta diferentes forças e fraquezas na avaliação da adequação do modelo fatorial confirmatório (Taylor e colaboradores, 2003). São eles: o Teste QuiQuadrado deve ser não-significativo; a razão entre quiquadrado e graus de liberdade deve ser menor que 5 (em valores nominais) ou, preferencialmente, menor que 2; o GFI deve apresentar um índice superior ou igual a 0,85 ; o AGFI deve apresentar um índice superior ou igual a 0,80 ; $\mathrm{e}$, finalmente, o RMS deve apresentar um índice inferior ou igual a 0,10 (Anderson \& Gerbing, 1984; Cole, 1987; Marsh, Balla \& McDonald ,1988).

Essa amostra apresentou três medidas de adequação ao modelo em desacordo com os critérios Psico-USF, v. 11, n. 2, p. 175-183, jul./de₹. 2006 padrões: qui-quadrado $\left(\chi^{2}(844, \mathrm{~N}=285)=2060,480 ; p<\right.$ $0,001)$, GFI $(0,724)$ e AGFI $(0,695)$, o que coloca em dúvida a adequação do modelo para a amostra. As últimas duas medidas de adequação ao modelo estão de acordo com os critérios padrões: $\chi^{2} / \mathrm{gl}(2,44)$ e RMS $(0,071)$.

Tendo em vista, por um lado, os resultados insatisfatórios obtidos na análise e, por outro, os resultados de outros estudos com o mesmo instrumento, resolveu-se realizar outras duas análises. A primeira dividiu o quarto fator em dois, de acordo com o conteúdo de seus itens (testando assim um modelo pentadimensional), uma vez que Barbosa e Balbinotti (2003) sugerem a não-compatibilidade entre os itens de conteúdo autocontrole e ações agressivas. A segunda análise retirou o quarto fator e testou a validade dos três fatores restantes (portanto, um modelo tridimensional), já que os dois estudos mencionados apontam problemas no quarto fator.

Nos dois casos observou-se melhora nos indicadores, ainda assim os importantes indicadores GFI e AGFI não foram satisfatórios. No caso do modelo pentadimensional obteve-se um qui-quadrado significativo $\left(\chi^{2}(859, \quad \mathrm{~N}=285)=1994,464 ; p<0,001\right)$, insatisfatórios GFI $(0,750)$ e AGFI $(0,723)$, adequada razão $\left(\chi^{2} / \mathrm{gl}=2,32\right)$ e RMS $(0,068)$. No caso do modelo tridimensional obteve-se um qui-quadrado significativo $\left(\chi^{2}{ }_{(495, \mathrm{~N}=285)}=1413,074 ; p<0,001\right)$, melhores, porém ainda insatisfatórios GFI $(0,757)$ e AGFI $(0,724)$, e adequada razão $\left(\chi^{2} / g 1=2,85\right)$ e RMS $(0,081)$.

\section{Discussão dos resultados}

Quanto aos resultados obtidos nas análises preliminares à consistência interna, são interpretados como satisfatórios, pois não houve aderência predominante (seja positiva ou negativa) em nenhum dos itens isolados ou das dimensões individualmente, com médias muito próximas aos valores extremos. Destaca-se, ainda, que a variabilidade dos resultados foi restrita, denotando-se, assim, certa homogeneidade na dispersão avaliada, independente do item ou dimensão estudada.

Observou-se uma grande variação nas correlações item-item, o que se interpretou como um fenômeno positivo, pois os pares pouco relacionados entre si são, fundamentalmente, compostos por um item de um fator e outro de outro fator. Inesperado seria, evidentemente, se existisse um índice de correlação extremamente fraco (ou inexistente) entre algum par de itens dentro de um mesmo fator, o que não ocorreu.

As correlações item-total obtiveram uma mediana de força moderada. Não foram encontradas correlações negativas, o que significa um excelente 
prognóstico na análise de correlações nessa escala. Esse prognóstico se confirma, por um lado, com as correlações item-dimensão, que se apresentaram bastante satisfatórias e, por outro lado, com os coeficientes alfa de Cronbach encontrados para as dimensões do IMCA ( $\alpha$ entre 0,71 e 0,89 ), que são perfeitamente comparáveis aos resultados dos outros $(\alpha$ entre 0,70 e 0,87 ) estudos.

No que diz respeito à análise fatorial, três modelos foram testados (modelos tri, tetra e pentadimencional). $\mathrm{O}$ indicador qui-quadrado significativo ( $p$ $<0,001)$, encontrado em todos os modelos testados, é tipicamente um resultado de grandes amostras (Cole, 1987; Marsh e colaboradores, 1988). Por se tratar de uma estatística extremamente sensível ao número de sujeitos da amostra, alguns autores têm descartado esse dado de suas análises.

Ainda assim, mesmo que esse resultado seja descartado, os demais resultados não permitem assegurar a adequação do modelo à amostra em nenhum dos três modelos testados. Pode-se notar que esses resultados estão de acordo com os encontrados em outras amostras de população de estudantes da Grande Porto Alegre (Barbosa \& Balbinotti, 2003; Balbinotti e colaboradores, no prelo), onde a escala mostrou-se satisfatoriamente fidedigna, porém nãoválida. Sendo assim, não se pode, com a amostra estudada, assumir a validade absoluta de nenhum dos modelos testados (modelos tri, tetra e pentadimencional). Os resultados sempre se apresentam miscigenados (alguns dados se aditam ao modelo, outros não).

\section{Conclusões}

Este estudo teve por objetivo verificar importantes qualidades métricas (consistência interna e modelo fatorial confirmatório) do IMCA-43 com estudantes da região metropolitana de Porto Alegre e comparar os resultados aqui obtidos com aqueles de outros estudos. Quanto às conclusões relativas à consistência interna, verificou-se que o IMCA é consistente, o que significa que as correlações interitens são, no mínimo, satisfatórias, ao menos para uso com os estudantes desta amostra. Destaca-se que não houve correlações negativas entre os pares de itens da escala total.

Quanto às conclusões relativas à análise fatorial confirmatória, verificou-se que o IMCA apresenta problemas de validade. Com exceção da análise da razão e do RMS, todas as outras três importantes medidas de adequação ao modelo (Qui-Quadrado, GFI e AGFI) estão em desacordo com os critérios padrões, de forma que não se pode garantir a adequação de nenhum dos três modelos testados (modelos tri, tetra e pentadimencional) para a amostra estudada.

Por fim, destacam-se algumas das importantes limitações desta pesquisa: não tratar-se de dados colhidos aleatoriamente (o que poderia, ao menos em parte, explicar o fenômeno da não-validade) e os adolescentes serem todos de escolas públicas (o que pode estar enviesando os resultados obtidos). Novos estudos deveriam dar conta dessas limitações e testar outras importantes propriedades métricas do IMCA-43, inclusive para outras populações específicas de adolescentes brasileiros (como os estudantes de escolas privadas, por exemplo).

\section{Referências}

Altshuler, J. L. \& Ruble, D. N. (1989). Developmental changes in children's awareness of strategies for coping with uncontrollable stress. Child Development, 60, 1337-1349.

Amirkhan, J. H. (1990). A factor analytically derived measure of coping: The coping strategy indicator. Journal of Personality and Social Psychology, 59, 1066-1074.

Anderson, J. C. \& Gerbing, D. W. (1984). The effect of sampling error on convergence, improper solutions, and goodness-os-fit indices for maximum likelihood confirmatory factor analysis. Psychometrika, 49, 155173.

Angers, M. (1992). Initiation pratique à la methodologie des sciences bumaines. Montréal: Les Éditions de la Chenelière.

Antoniazzi, A. S. (2000). Desenvolvimento de instrumentos para a avaliação de coping em adolescentes brasileiros. (Tese de Doutorado). Porto Alegre-RS: Universidade Federal do Rio Grande do Sul - Instituto de Psicologia, Curso de Pós-Graduação em Psicologia do Desenvolvimento.

Antoniazzi, A. S., Dell'Aglio, D. D. \& Bandeira, D. R. (1998). A evolução do conceito de coping: uma revisão teórica. Temas de Psicologia, 3, 273-294.

Bagby, R. M., Taylor, G. J. \& Parker, J. D. (1992). Reliability and validity of the 20-Item TorontoAlexithymia-Scale. Pôster apresentado no Encontro do $50^{\circ}$ Aniversário da American Psychosomatic Society. Nova York.

Balbinotti, M. A. A., Barbosa, M. L. L. \& Wiethaeuper, D. (no prelo). Estudos da consistência interna e fatorial confirmatório do IMCA-43 com estudantes brasileiros. Revista Psi. 
Band, E. B. \& Weisz, J. R. (1988). How to feel better when it feels bad: Children's perspectives on coping with everyday stress. Developmental Psychology, 24, 247253.

Barbosa, M. L. L. \& Balbinotti, M. A. A. (2003). Estudo das qualidades métricas de uma medida de coping para adolescentes (Manuscrito não-publicado). São Leopoldo, UNISINOS, Centro de Ciências da Saúde.

Berry, J. W., Poortinga, Y. H., Segall, M. H. \& Dasen, P.R. (1992). Cross-cultural psychology: Research and applications. Cambridge (UK): Cambridge Univ. Press.

Briggs S. R. \& Cheek J. M. (1986). The role of factor analysis in the development and evaluation of personality scales. Journal of Personality, 54, 6-48.

Bryman, A. \& Cramer, D. (1999). Quantitative data analysis: A guide for social scientists. New York: Routledge.

Carver, C. S., Scheier, M. F. \& Weintraub, J. K. (1989). Assessing coping strategies: A theoretically based approach. Journal of Personality and Social Psychology, 56, 267-283.

Clark, K. A., Bormann, C. A., Cropanzano, R. S. \& James, K. (1995). Validation evidence for three coping measures. Journal of Personality Assessment, 65, 434-455.

Cole, D. A. (1987). Utility of confirmatory factor analysis in test validation research. Journal of Consulting and Clinical Psychology, 55, 584-594.

Compas, B. E., Banez, G. A., Malcarne, V. \& Worsham, N. (1991). Perceived Control and Coping with stress: A developmental perspective. Journal of Social Issues, 47, 23-34.

Costa P. T. Jr., Somerfield, M. R. \& McCrae, R. R. (1996). Personality and coping: A reconceptualization. Em M. Zeidner \& N. S. Endler (Orgs.). Handbook of coping: Theory, research, applications (pp. 44-61). New York: Wiley.

Cronbach, L. J. (1951). Coefficient alpha and the internal structure of tests. Psychometrika, 16, 297-334.

Cronbach, L. J. (1988). Initernal-consistency of tests: Analyses old and new. Psychometrika, 53, 63-70.

Croncbach, L. J. (1989). Construct validation after thirty years. Em R. L. Linn (Ed.). Intelligence: Measurement, theory and public policy - Proceedings of a symposium in honor of Lloyd G. Hamphreys. Chicago, IL: University of Chicago Press.

Cronbach, L. J. (1996). Fundamentos da testagem psicológica. Porto Alegre, RS: Artes Médicas.
Cronbach, L. J. \& Meehl, P. E. (1955). Construct validity in psychological tests. Psychological Bulletin, 52, 281-302.

Cronbach, L. J., Rajaratnam, N. \& Gleser, G. C. (1963). Theory of generalizability: A liberalization of reliability theory. The British Journal of Statistical Psychology, 16(2), 137-163.

Croyle, K. L., Troester, A. I., Fields, J. A., StraitsTroester, K. A., Lyons, K. E. \& Pahwa, R. (2003). Presurgical coping, depression, and quality of life in persons with Parkinson's disease. Journal of Clinical Psychology in Medical Settings, 10(2), 101-107.

Darnall, B. D. (2002). The relationship between athletic identity, elite athlete self-esteem, coping, and pathology in elite athletes. Dissertation Abstracts International: Section B: The Sciences and Engineering, 63(6B), 30-43.

Dassa, C. (1999). Analyse multidimensionnelle exploratoire et confirmative. Montreal: Univesité de Montreal.

Dell'Aglio, D. (2000). O processo de coping, institucionalização e eventos de vida em crianças e adolescentes. (Tese de Doutorado) Porto Alegre-RS: Universidade Federal do Rio Grande do Sul - Curso de PósGraduação em Psicologia do Desenvolvimento.

Dell'Aglio, D. \& Hutz, C. S. (2002). Estratégias de coping e estilo atribucional de crianças em eventos estressantes. Estudos de Psicologia da Universidade Federal do Rio Grande do Norte, 7(1), 5-13.

Endler, N. S. \& Parker, J. D. A. (1990). Multidimensional assessment of coping: A critical evaluation. Journal of Personality and Social Psychology, 58, 844-854.

Endler, N. S. \& Parker, J. D. A. (1999). Coping Inventory for Stressful Situations (CISS). Manual (2nd. ed.). Toronto: Multi-Health Systems Inc.

Endler, N. S., Summerfledt, L. J. \& Parker, J. D. A. (1998). Coping with health problems: Developing a reliable and valid multidimensional measure. Psychol. Assess., 10, 195-205.

Epstein, S. (1990). Cognitive-experiential self-theory. In L. A. Pervin (Ed.). Handbook of personality: Theory and research (pp. 165-192). New York: The Guilford Press.

Folkman, S. \& Lazarus, R. S. (1980). An analysis of coping in a middle-aged community sample. Journal of Health and Social Behavior, 21, 219-239.

Folkman, S. \& Lazarus, R. S. (1985). If it changes it must be a process: A study of emotion and coping during three stages of a college examination. Journal of Personality and Social Psychology, 48, 150-170. 
Folkman, S., Lazarus, R. L., Dunkel-Schetter, C., DeLongis, A. \& Gruen, R. (1986). Dynamics of a stressful encounter: Cognitive appraisal, coping, and encounter outcomes. Journal of Personality and Social Psychology, 50, 992-1003.

Garson, D. (2005). PA 765 Statnote: An online textbook. Disponível em: <http://www2.chass.ncsu.edu/garson/ pa765/ statnote.htm>. Acesso em: 6.mar.2005.

Gayton, J. E. (2002). Working models of attachment and health threats: Distress, appraisal, coping and health-related behaviours in colorectal cancer. Dissertation Abstracts International: Section B: The Sciences and Engineering, 63(1-B), 524.

Gimenes, M. G. G. (1997). A teoria do enfrentamento e suas implicações para sucessos e insucessos em psiconcologia. Em M. G. G. Gimenes \& M. H. Fávero (Orgs.). A mulher e o câncer (pp. 111-147). Campinas: Psy.

Gimenes, M. G. G. \& Queiroz, B. (1997). As diferentes fases de enfrentamento durante o primeiro ano após a mastectomia. Em M. G. G. Gimenes \& M. H. Fávero (Orgs.). A mulher e o câncer (pp. 171-195). Campinas: Psy.

Heckhausen, J. \& Schulz, R. (1995). A lifespan theory of control. Psychological Review, 102, 284-304.

Holahan, C. J. \& Moos, R. H. (1985). Life stress and health: Personality, coping, and family support in stress resistance. Journal of Personality and Social Psychology, 49, 739-747.

Holahan, C. J., Moos, R. H. \& Schaefer, J. A. (1996). Coping, stress, resistance, and growth: Conceptualizing adaptive functioning. Em M. Zeidner \& N. S. Endler (Orgs.). Handbook of coping: Theory, research, applications (pp. 24-43). New York: Wiley.

Hussain, F. A. \& Cochrane, R. (2003). Living with depression: coping strategies used by South Asian women, living in the UK, suffering from depression. Mental Health, Religion and Culture, 6(1), 21-44.

Johnson, M. (2004). Approaching the salutogenesis of sense of coherence: The role of "active" self-esteem and coping. British Journal of Health Psychology, 9(3), 419-432.

Lazarus, R. S. \& Folkman, S. (1984). Stress, appraisal, and coping. New York: Springer.

Lee, J. M. (2004). Examining the risks of restraint: The relation between self-esteem and coping to disinhibited eating behavior. Dissertation Abstracts
International: Section B: The Sciences and Engineering, 64(8B), 40-47.

Lefkowitz, E. T. (2003). Predicting adjustment to college: A model of personality, coping strategies and college adjustment. Dissertation Abstracts International: Section B: The Sciences and Engineering, 64(6-B), 29-72.

Lopez, D. F. \& Little, T. D. (1996). Children's actioncontrol beliefs and emotional regulation in the social domain. Developmental Psychology, 32, 299-312.

Marsh, H. W., Balla, J. R. \& McDonald, R. P. (1988). Goodness-of-fit indexes in confirmatory factor analisys: The effect of sample size. Psychological Bulletin, 103, 391-410.

Nunnally, J. C. (1978). Psychometric Theory (2 $2^{\mathrm{a}}$ ed.). New York: McGraw-Hill.

Parker J. D. A. \& Endler, N. S. (1996). Coping and defense: A historical overview. Em M. Zeidner \& N. S. Endler (Orgs.). Handbook of coping: Theory, research, applications (pp. 3-23). New York: Wiley.

Parker, J. D. A., Endler, N. S. \& Bagby, R. M. (1993). If it changes it might be unstable: Examining the factor structure of the Way of Coping Questionnaire. Psychological Assessment, 5, 361-368.

Pestana, M. H. \& Gageiro, J. G. (2003). Análise de dados para ciências sociais: a complementaridade do SPSS ( $3^{\mathrm{a}}$ ed.). Lisboa: Silabo.

Pinheiro, F. A., Tamayo, M. R. \& Tróccoli, B. T. (2000). Mensuração de coping no ambiente ocupacional: tradução e validação da Escala de Lateck. [Resumo] Em Sociedade Brasileira para o Progresso da Ciência (Org.). Anais da 52a. Reunião Anual da SBPC. Brasília: SBPC, 235.

Reis, E. (2001). Estatística multivariada aplicada (2a ed.). Lisboa: Silabo.

Rodríguez-Marín, J., Terol, M. C., López-Roig, S. \& Pastor, M. (1992). Evaluación del afrontamiento del estrés: propriedades psicométricas del cuestionario de formas de afrontamiento de acontecimientos estresantes. Revista de Psicología de la Salud, 4, 59-82.

Savóia, M. G., Santana, P. R. \& Mejias, N. P. (1996). Adaptação do inventário de estratégias de coping de Folkman e Lazarus para o português. Psicologia USP, 7, 183-201.

Schwarzer, R. \& Schwarzer, C. (1996). A critical survey of coping instruments. Em M. Zeidner \& N. S. Endler (Orgs.). Handbook of coping: Theory, research, applications (pp. 107-132). New York: Wiley. 
Seidl, E. M. F., Tróccoli, B. T. \& Zannon, C. M. L. C. (2001). Análise fatorial de uma medida de estratégias de enfrentamento. Psicologia: Teoria e Pesquisa, 17(3), 225-234.

Taylor, G. J., Bagby, R. M. \& Parker, J. D. A. (2003). The Twenty - Item Toronto Alexithymia Scale IV. Reliability and factorial validity in diferent languages and cultures. Journal of Psichosomatic Research, 55, 277283.

Tucker, S., Brust, S. \& Richardson, B. (2002). Validity of the Depression Coping Self-Efficacy Scale. Archives of Psychiatric Nursing, 16(3), 125-133.

Vaillant, G. E. (1994). Ego mechanisms of defense and personality psychopathology. Journal of Abnormal Psychology, 103, 44-50.

Vitalino, P. P., Russo, J., Carr, J. E., Maiuro, R. D. \& Becker, J. (1985). The ways of coping checklist: Revision and psychometric properties. Multivariate Behavioral Research, 20, 3-26.

Wang, W. \& Wang, Y. (2003). Personality, coping style, and the SCL-90 results of medical students. Chinese Mental Health Journal, 17(2), 118-119.

Watkins D. (1989) The role of confirmatory factor analysis in cross-cultural research. International Journal of Psychology, 24, 685-701.
Wearden, A., Cook, L. \& Vaughan-Jones, J. (2003). Adult attachment, alexithymia, symptom reporting and health-related coping. Journal of Psychosomatic Research, 55(4), 341-347.

Weisz, J. R., McCabe, M. \& Denning, M. D. (1994). Primary and secondary control among children undergoing medical procedures: Adjustment as a function of coping style. Journal of Consulting and Clinical Psychology, 62, 324-332.

Willebrand, M. (2003). Coping, personality and cognitive processes in burn injured patients. Nordic Journal of Psychiatry, 57(3), 241-242.

Windover, A. K. (2002). The roles of dispositional coping, personality, and situational characteristics in situational coping behavior. Dissertation Abstracts International: Section B: The Sciences and Engineering, 63(1B), 555 .

Ying, P., Wen-Bin, G. \& Guo-Qiang, W. (2003). A study on relationship among depression coping styles and personality in major depression. Chinese Journal of Clinical Psychology, 11(3), 230-231.

Recebido em abril de 2005 Reformulado em abril de 2006 Aprovado em agosto de 2006

Sobre os autores:

Marcos Alencar Abaide Balbinotti é PhD em Psicologia pela Universidade de Montreal, no Canadá, com PósDoutorado realizado no Laboratório de Variáveis Afetivas. Desenvolve estudos em Desenvolvimento de Carreira, Variáveis Relacionadas à Escolha Profissional, Educação à Carreira, Psicologia do Trabalho, Psicometria. É pesquisador do Centro de Pesquisas Interuniversitárias sobre a Educação e a Vida no Trabalho, órgão canadense; professor pesquisador do Mestrado em Ciências da Saúde (Unisinos) e Ciências do Movimento Humano (UFRGS) e coordenador do Núcleo de Orientação Vocacional da Universidade do Vale do Rio dos Sinos (Unisinos).

Marcus Levi Lopes Barbosa é psicólogo formado pela Universidade do Vale do Rio dos Sinos (Unisinos) e mestrando do Programa de Pós-Graduação em Ciências do Movimento Humano, na Universidade Federal do Rio Grande do Sul (UFRGS).

Daniela Wiethaeuper é PhD em Psicologia Clínica e Dinâmica pela Universidade de Montreal, no Canadá. Desenvolve estudos e pesquisas em Psicoterapia, Variáveis Relacionadas a Estilos Língüísticos e Comnlementaridades Fstilísticas. além de trabalhos de validacão de instrumentos de nesauisa na área clínica. É inculada ao Grupo 
